\title{
Distributed Coordinated in-Vehicle Online Routing Using Mixed-Strategy Congestion Game
}

\author{
Lili $\mathrm{Du}^{1}$, Lanshan Han ${ }^{2}$, Xiangyang $\mathrm{Li}^{3}$
}

\begin{abstract}
This study proposes a coordinated online in-vehicle routing mechanism for smart vehicles with real-time information exchange and portable computation capabilities. The proposed coordinated routing mechanism incorporates a discrete choice model to account for drivers' behavior, and is implemented by a simultaneouslyupdating distributed algorithm. This study shows the existence of an equilibrium coordinated routing decision for the mixed-strategy routing game and the convergence of the distributed algorithm to the equilibrium routing decision, assuming individual smart vehicles are selfish players seeking to minimize their own travel time. Numerical experiments conducted based on Sioux Falls city network indicate that the proposed distributed algorithm converges quickly under different smart vehicle penetrations, thus it possesses a great potential for online applications. Moreover, the proposed coordinated routing mechanism outperforms traditional independent selfish-routing mechanism; it reduces travel time for both overall system and individual vehicles, which represents the core idea of Intelligent Transportation Systems (ITS).
\end{abstract}

Keywords: Online coordinated in-vehicle routing guidance, Routing game, Distributed algorithm, Discrete choice model

\section{Introduction and Motivation}

Recent years, wireless communication, on-board computation facilities (personal digital assistants, smart phones, etc) and advanced sensor techniques (loop detector, camera, GPS-based vehicle probe,etc) have been integrated into transportation systems. These new technologies establish information exchange in vehicle-tovehicle (V2V) and vehicle-to-infrastructure (V2I) networks, and further enable real-time traffic information to be collected, processed, and disseminated among smart vehicles, road infrastructure, as well as traffic management centers. Accordingly, a type of well-connected and information-rich transportation systems, connected vehicle system (CVS), is under rapid development and is expected to be fully implemented in the near future. It enables us to create distributed but coordinated traffic applications to improve mobility, safety, environmental friendliness of transportation systems.

The proposed research is particularly interested in improving traffic mobility through CVS technology. We notice that CVS has prompted many advanced in-vehicle routing guidance systems embedded with a-priori or en-route algorithms $[15,2,8,10,11,2]$. Some of them have been implemented by pioneer practitioners, such as Waze, a community-based traffic and navigation app developed by Israeli start-up Waze Mobile. These routing guidance systems seek to intelligently guide individual travelers to avoid traffic congestion $[12,7,13,14,16,10,5]$, taking advantage of the real-time traffic information provided to individual smart vehicles.

Even though CVS has been granted a great potential to intelligently route travelers (smart vehicles), scholars have recognized that if each smart vehicle independently chooses the shortest path based on uniformly shared real-time traffic information, it may only be beneficial when smart vehicles are the minority

\footnotetext{
${ }^{1}$ Corresponding author, Department of Civil, Architectural and Environmental Engineering, Illinois Institute of Technology, 3201 South Dearborn Street, Chicago, Illinois 60616 U.S.A., Email: ldu3@iit.edu

${ }^{2}$ Nielsen Marketing Analytics, 1603 Orrington Ave. Suite 1900, Evanston, Illinois 60201, U.S.A., Email: lanshanhan@gmail.com

${ }^{3}$ Department of Computer Science, Illinois Institute of Technology, 10, West 31st Street, Chicago, Illinois 60616 U.S.A., xli@cs.iit.edu
} 
and their route choices do not impact traffic flow significantly. Smart vehicles may take advantages of the real-time information and find shorter paths which non-smart vehicles may not be able to recognized. However, as smart vehicles become the majority (which is expected as the related technologies are improved further), their route choices will impact traffic flow significantly. Then, current uniform real-time information provision may lead to even worsen traffic congestion, given each smart vehicle still selfishly and independently chooses its own shortest paths. For example, many smart vehicles sharing uniform information are very likely to choose a same link/corridor not crowed at the time that route choices are made, and then it becomes highly congested when they arrive at the link/corridor.

This inherent deficiency of current routing methods with uniform real-time provision is rooted from the inconsistency between system performance (system-optimality) and individual vehicles' route choice behavior (user-optimality). As different strategies are used to treat this inconsistency, various routing mechanisms can be developed. This study considers most of current in-vehicle routing mechanisms as independent selfishrouting mechanism (IRM), which gives the priority of the routing to user-optimality and leaves system performance out of control or under very limited control. As we mentioned before, IRM may cause system congestion when adopted by majority of the traffic flow. Accordingly, individual users also lose the benefit of using it. On the other hand, if the priority of routing mechanism is given to system-optimality, pure systematical optimal routing mechanisms (SRM) can be developed, which sacrifice some vehicles' travel time to achieve system optimality (i.e. smart vehicles are fully cooperated). SRM overwhelms the need of user-optimality and certainly conflicts the selfish nature of vehicles' route choice behavior, thus cannot work well in practice. The above observation indicates a need of new routing strategies/mechanisms which enable proper coordinations among vehicles (i.e. individual vehicles and traffic system) so that both the overall system and individual vehicles benefit from the provision of real-time traffic information. Recent advances of CVS have sustained the technique feasibility to develop this kind of coordination among smart vehicles, and to implement coordinated routing mechanisms.

Motivated by the above views, the proposed study seeks to develop a novel coordinated online in-vehicle routing mechanism (CRM) based upon routing game theory and distributed algorithm technique. The proposed CRM aims to artfully overcome the deficiency of traditional IRM without violating selfish natural of route choice behavior. Under this mechanism, we consider a trip of a smart vehicle from its origin to destination as a constant information exchange and multiple route choice decision process. Namely, during a trip, each individual vehicle keeps collecting and disseminating traffic information including its preference on the route options. As the trip is close to each intersection, a smart vehicle joins an online routing coordination group and decides route choice in candidate route options. The coordination group is dynamically formed by the vehicles that are close to an intersection and/or about to make routing decisions in the traffic network under consideration. The priorities of its candidate route options (route choice probability distributions) are identified by near future traffic condition revealed by recent traffic condition and route choices of all other smart vehicles in the coordination group. In an online routing coordination group, each smart vehicle iteratively proposes and updates its routing choice priorities, responding to real-time traffic information (incorporating their most recent route choices) based on exchanging information with other smart vehicles through an online communication environment; the negotiation process ends with a resolution (represents an equilibrium route choice decision) among all smart vehicles, which leads to the final route choice of smart vehicles. The proposed mechanism guarantees the optimality of individual vehicle's route choice priorities (given others in the group do not deviate from their priorities). And as will be shown in the experiments, this mechanism will also lead to much better overall system performance compared to pure selfish-routing without coordination.

The proposed mechanism has four distinguished technical characteristics. (i) The proposed routing mechanism can be regarded as an adaptive online routing process. Smart vehicles re-decide route choices at each intersection according to real-time traffic information. (ii) The routing mechanism relies on information sharing. Hence, a virtual online routing coordination group and an underlying supporting communication platform are needed. They are enabled by V2V and V2I networks in CVS. The privacy issues associated with this information sharing can be solved by privacy protection algorithms such as [9]. (iii) The routing decision of each vehicle is the priorities of the candidate route options, which is represented by a probability distribution. The combination of traffic information sharing and routing decision is reflected by modeling the route decision making process as a mixed strategy routing game. The equilibrium routing decision represents user-optimal route decision for each individual vehicle. (iv) The coordinated routing mechanism 
is proposed to be implemented in a distributed fashion by employing the in-vehicle computation resources. It implies that each vehicle will carry out the computation locally and a super powerful central control unit is not needed. In short, the aforementioned characteristics indicate that the proposed routing mechanism belongs to adaptive distributed online routing strategy; each routing decision making procedure is modeled as a mixed strategy atomic routing game; and it is proposed to be distributively implemented. Even though this proposed routing mechanism is initiated by the case of high smart vehicle penetrations and it shows more value to be applied under this condition, this study will also demonstrate that it works reasonably well under low smart vehicle penetrations. Throughout the paper, we will exchangeably use "smart vehicles" and "travelers" to adapt the need of proper articulation in the context.

The rest of this paper is organized by this structure. Section 2 reviews existing relevant literature. Section 3 proposes the coordinated online in-vehicle routing mechanism. The existence and uniqueness of an equilibrium routing decision among the smart vehicles in a coordinated routing group are proved. Section 4 develops a simultaneously updating distributed algorithm; its convergence is established. Section 5 conducts numerical experiments to demonstrate the applicability of the proposed approach. Section 6 concludes the whole paper.

\section{Literature Review}

The proposed research aims to develop an online in-vehicle routing mechanism employing real-time traffic information, which seeks to find optimal route choice decision for individual vehicles and to avoid system traffic congestion as well. In literature, this research sits in the field of real-time in-vehicle routing guidance development. In the literature, we can find some related existing work, which can be classified into reactive and anticipatory categories [18]. The following will review the closest work and differentiate the proposed studies from them as well.

Reactive guidance systems $[2,8,15]$ provide route guidance based on a snapshot of the traffic condition at that time without including traffic predictions. Most of existing reactive in-vehicle routing methods are in an independent selfish fashion, thus they cannot avoid system congestion when a high penetration of smart vehicles is involved. Among the cited literature, we noticed that [8] aims to find a routing algorithm which balances individual travelers' selfish route choice behavior and system performance, which represents a similar idea to this study. More exactly, [8] develops a system optimal routing approach, which explicitly integrated user behavior constraints and leads to a better system performance than user equilibrium. Hence, the key idea stays with using central control rather than building up spontaneous coordination among smart vehicles, which is different to this study. Using central control for in-vehicle routing requires very powerful centralized computation resources to carry out the computation load as a big number of vehicles are involved, and hence is not practical for real-time applications. In addition, the reliability of this kind of system needs to be carefully evaluated and backup resources need to be deployed to avoid system failure. Overall, one of the advantages of reactive guidance is that it can respond quickly to demand changes or incidents. The proposed routing mechanism shares this common advantage, providing routing suggestions based on up-to-date traffic information, but differentiates itself from them by artfully integrating coordination among vehicles in the routing decision process.

Anticipatory guidance systems, such as $[2,10,11]$ and many references wherein, predicted future demands and traffic conditions and then provided routing recommendations accordingly. Traffic forecasting, the critical component in these systems, is produced by a traffic assignment procedure, which takes into account of the vehicle behavior: seeking user-optimal route choices. Among all the existing efforts in this category, the work most closely to this study is developed by [10]. Based on simulator INTEGRATION, [10] implemented a process, which iteratively performs traffic flow forecasting and traffic assignment. When the iteration terminates with a fixed point assignment (i.e. the new forecasting is sufficiently close to the old one), useroptimality is achieved. Even though the proposed study have some common ideas shared with [10], such as attempt to balance user-optimality and system performance, the underlying methodologies are substantially different in the following aspects. 1) If we consider the iterative traffic forecasting and assignment in [10] equivalent to routing coordinations between vehicles, [10] conducted this coordination in a centralized fashion (dynamic traffic assignment in the network) instead of the distributed fashion proposed in this research. 2) [10] mainly relays on simulation results to demonstrate the efficiency of their routing guidance as well as the impact of penetrations. Some important questions, such as whether the procedure can terminate, what kind 
of fixed point assignment will the procedure end up with, have not been answered by rigorous mathematical arguments, while the proposed study develops mathematical analysis to the existence, uniqueness of the fixed point (user-optimality) in CRM, and the convergence of the distributed algorithm to implement CRM. 3) [10] indicates anticipatory routing brings in more benefit when link-exit travel time estimation is employed. However, it also represents a deficiency of real-time anticipatory routing. Namely, when all anticipatory vehicles with the same destination reaches the same node in a single time period, it is very hard to synthesize many link-exit travel time estimations into one forecasting in that time period. This deficiency in turn motivates the proposed study, which aims to address the traffic congestion resulting from routing all vehicles with same destination to a single link.

The state of the art indicates that most of previous studies develop in-vehicle routing guidance with the underlying logic: individual vehicles seeking routes independently without coordinating with other vehicles. This may result from the lack of reliable communication technologies from vehicles to vehicles or from vehicles to infrastructure previously. However, these technology obstacles have been cleared up. A new wellconnected transportation system is gradually emerging. Thus, it is the time to develop a new generation of online routing guidance to improve both the network performance and individual vehicles' satisfaction, incorporating the available communication capability. This paper aims to introduce a routing mechanism that achieves this goal and also partially establishes the theoretical foundations of coordinated routing mechanisms. By applying game theoretical approaches as well as convergence analysis in optimization theory, we demonstrate that it is possible to allow smart vehicles to 1) coordinate in route choice decision making, and 2) utilize their own computational power instead of relying completely on a central control unit. We shall show that under certain conditions, the coordination will end up with a resolution to all the vehicles, and this resolution can be found in a distributed fashion. The proposed research also represents an initial effort on introducing distributed algorithms into transportation systems. As more and more smart devices are deployed into transportation networks, distributed algorithms, which have found application in many areas, will surely find more applications in traffic control and operation. Overall, the proposed study is of significant theoretical and practical importance for creating a smarter transportation system.

\section{Coordinated Routing Mechanism}

This study considers that there are a large volume of smart vehicles moving in the network. At a given short time period, there is a group of smart vehicles which need to make route choice decisions among a number of candidate routes, according to the latest real-time traffic information. The routing decision is made by first determining the priorities of candidate routes (a probability distribution), and then picking a route based on the priorities. To address this online in-vehicle routing problem, this study proposes CRM, a coordinated online in-vehicle routing mechanism, and models it as a mixed strategy routing game, in which the process that smart vehicles decide their own route choice priorities is treated as a negotiation and coordination process among smart vehicles. These vehicles spontaneously form a routing coordination group according to recent routing decision requests. In the routing coordination group, each smart vehicle acts as a player, seeking to find the best online route choice priority, which leads to the probabilities of choosing the candidate paths with minimum expected travel time. The coordinated vehicles iteratively update and propose their routing choice priority in responding to their evaluation of near future traffic condition based on shared online traffic information among smart vehicles through an online communication environment. The negotiation process repeats several iterations $(t)$ until all travelers accept and won't change their route choice priorities (i.e. an equilibrium route choice priority decision (ERD)). Then traveler $\nu$ will pick a route based on that. During the trip of each traveler, a number of route decisions following the CRM can be made until reaching the destination.

The rest of this section includes technical details about the CRM regarding traffic condition evaluation and route choice priority proposing. Furthermore, this section proves the existence and uniqueness of ERD for the proposed mixed strategy routing game. This proposed CRM can be implemented through a distributed algorithm which will be introduced in Section 4.

\subsection{Mathematical notations}

We consider a transportation network represented by a directed graph $G(N, A)$ with $N$ being the set of nodes and $A$ being the set of arcs. Let the number of arcs be $l=|A|$ and the number of nodes be 
$n=|N|$. Suppose there are $m$ smart vehicles in a coordination routing group, labeled by $\nu=1, \cdots, m$, equipped with wireless communication and onboard computation equipments traversing the network. At the moment of making route choice decisions, each vehicle $\nu$ has a specific origin-destination (OD) pair given by $\left(r_{\nu}, s_{\nu}\right) \in N \times N$ with a set of $k^{\nu}$ possible candidate paths connecting them, denoted as $\mathcal{H}^{\nu} \triangleq\left\{h^{\nu, i}\right\}_{i=1}^{k^{\nu}}$. Define $k=\sum_{\nu=1}^{m} k^{\nu}$ to be the total number of paths under consideration in $G$. Let $f_{\ell}$ represent the expected traffic volume on link $\ell$, and $f^{\nu, i}$ represent the expected traffic volume on path $i$ connecting the OD pair of vehicle $\nu$. Accordingly, $\Omega^{\nu} \in \mathbb{R}^{k^{v} \times l}$ represents the path link incidence matrix for vehicle $\nu ; \Omega=\left[\Omega^{\nu}\right]_{\nu=1, \cdots, m}$ is formed by stacking up $\Omega^{\nu}$ for all the vehicles $\nu$. $\Omega$ represents path link matrix over the whole network and all vehicles, i.e., for each element $\delta_{\nu, i}^{\ell}$ in $\Omega$ we have

$$
\delta_{\nu, i}^{\ell}=\left\{\begin{array}{ll}
1 & \text { if } \ell \in h^{\nu, i} \\
0 & \text { otherwise }
\end{array} .\right.
$$

The link travel cost is denoted by $c_{\ell}$, which, in this research, is assumed to be a function of the link traffic volume, and hence is written as $c_{\ell}\left(f_{\ell}\right)$ sometime. Throughout the paper, we assume:

(A) The link travel time function $c_{\ell}\left(f_{\ell}\right)$ is continuously differentiable and strictly increasing in $f_{\ell}$. Moreover, the derivative of the link travel time function, $c_{\ell}^{\prime}\left(f_{\ell}\right)$ is an increasing function of $f_{\ell}$.

Assumption (A) captures the properties that link travel time increases as the link flow increases, and the increasing rate also increases as the link becomes more crowded. These properties are satisfied by different travel time functions, such as the widely used BPR function. The travel time of path $i$ for vehicle $\nu$ is denoted as $C^{\nu, i}$. Let $C$ represent the set of path travel times for all vehicles. The route choice priority of traveler $\nu$ is represented by a discrete probablity distribution: $\mathcal{P}^{\nu} \triangleq\left\{p^{\nu, i}\right\}_{i=1}^{k^{\nu}}$, where $p^{\nu, i}$ represents the probability that vehicle $\nu$ chooses path $h^{\nu, i}$, and $\sum_{i=1}^{k^{\nu}} p^{\nu, i}=1$. We can see that $f^{\nu, i}=p^{\nu, i}$ for all $\nu=1, \cdots, m$ and $i=1, \cdots, k^{\nu}$, therefore, sometime they are used exchangeably int his paper. Let $\mathcal{P}$ represents the set of route choice priorities/distributions of all travelers. Note that two vehicles may have a same OD pair.

We assume that the vehicles are aware of the real-time information below: (a) the real-time link traffic flow information; (b) the real-time other vehicles' route choice priorities. Both information (a) and (b) can be obtained through the communication between vehicle-to-vehicle $(\mathrm{V} 2 \mathrm{~V})$ or vehicle to infrastructure (V2I) (such as traffic information management center). This study considers that the traffic information is aggregated and processed perfectly without accuracy and time delay issues.

\subsection{Evaluating traffic condition}

In the routing coordination group, individual vehicles share real-time traffic information and their route choice priorities, and then evaluate traffic condition in the near future. Namely, At iteration $t$, individual vehicle will predict the expected traffic flow $f_{t}^{\ell}$, which is calculated by the latest traffic flow information and other vehicles' route choice proposals. Considering traveler $\nu$ choosing link $\ell$ at iteration $t$ as a discrete random variable $y_{t}^{\nu, \ell}$, we have

$$
y_{t}^{\nu, \ell}=\sum_{h^{\nu, i} \ni \ell} p_{t}^{\nu, i}
$$

i.e., the sum of the probabilities that the paths chosen by traveler $\nu$ include link $\ell$. The expected number of travelers choosing link $\ell$ equals to $\sum_{\nu} y_{t}^{\nu, \ell}$. Furthermore, the expected traffic flow on link $\ell$ from this routing decision group is given by

$$
\begin{aligned}
f_{t}^{\ell} & =E\left[\sum_{\nu=1}^{m} \sum_{h^{\nu, i} \ni \ell} p_{t}^{\nu, i}\right] \\
& =\sum_{\nu=1}^{m} \sum_{i=1}^{k^{\nu}} p_{t}^{\nu, i} \delta_{\nu, i}^{\ell} .
\end{aligned}
$$

Note that link traffic flow may also include vehicles outside of this coordination group, denoted by $f_{\ell}^{b}$. We assume that $f_{\ell}^{b}$ can be obtained by the information shared by certain vehicles in the coordination group. Hence, it be available to all the vehicles in the group due to information exchange. Moreover, we consider $f_{\ell}^{b}$ as a constant value (i.e. a kind of "background" flow) during the short routing decision making process. Namely, it does not change as the decision process goes on. Thus, we don't explicitly include this flow in the 
travel cost function $c_{\ell}$ to simply the notation and articulation. Accordingly, the expected travel time on link $\ell$ equals to $c_{\ell}\left(f_{t}^{\ell}\right)$, where $c_{\ell}$ is the link travel (cost) function. Throughout this paper, we assume that $c_{\ell}\left(f_{\ell}\right)$ is continuous and strictly increasing in $f_{\ell}$ for all $\ell=1, \cdots, l$. (BPR function applied in this study satisfies this condition). The expected travel time of path $h^{\nu, i}$ at time $t$ will be re-evaluated through

$$
C_{t}^{\nu, i}=\sum_{\ell \in h^{\nu, i}} c_{\ell}\left(f_{t}^{\ell}\right)
$$

\subsection{Proposing route choice}

Once aware of new traffic condition, each smart vehicle $\nu$ will computes its new targeting route choice priority $\underset{t+, 1}{\overrightarrow{t+i}}$ through a multinomial logit (MNL) choice model [17] in Equation (3) below:

$$
\underset{p_{t+1}, \nu, i}{\sum_{i=1}^{\kappa^{\nu}} e^{-V_{\nu, i}}} \triangleq \mathbf{p}^{\nu, i}\left(p_{t}\right) \forall \nu=1, \cdots, m ; i=1, \cdots, k^{\nu},
$$

where $V_{\nu, i}=\alpha^{\nu}+\beta^{\nu} C_{t}^{\nu, i}$ represents the utility of path $h^{\nu, i} ; \alpha^{\nu}$, and $\beta^{\nu}$ are positive constant value and $C_{t}^{\nu, i}$ is the expected travel time of path $h^{\nu, i}$ at iteration $t$, and $p_{t} \triangleq\left(p^{\nu, i}\right)_{\nu=1, \cdots, m ; i=1, \cdots, k^{\nu}}$ is the vector of the probabilities on all the paths for all the vehicles at iteration $t ; \mathbf{p}=\left\{\mathbf{p}^{\nu, i}\right\}_{\nu=1, \cdots, m ; i=1, \cdots, k^{\nu}}$ represents the map from $p_{t}$ to $p_{t+1}$.

Next, a new route choice priority $p_{t+1}^{\nu, i}$ is obtained through taking a convex combination of the targeting route choice priority and its current route choice priority $p_{t}^{\nu, i}$ using a certain step length. As we will see in Section 4, this step length is introduced to make sure the convergence of the whole route choice negotiation process.

\subsection{Equilibrium routing decision}

As aforementioned, updating traffic condition and proposing new route choice priority will keep going until the targeting route distribution is the same as the current route choice priority for all the vehicles, in which situation, we say that an equilibrium routing decision has been reached. Mathematically, the equilibrium route choice decision is a joint probability $p_{*}=\left(p_{*}^{\nu, i}\right)_{\nu=1, \cdots, m ; i=1, \cdots, k^{\nu}}$ such that:

$$
\mathbf{p}\left(p_{*}\right)=p_{*},
$$

or in another word, is a fixed point of the map $\mathbf{p}$, where

$$
\mathbf{p}(p)=\left(\mathbf{p}^{\nu, i}(p)\right)_{\nu=1, \cdots, m ; i=1, \cdots, k^{\nu}} .
$$

Then, a natural question is: does an ERD among smart vehicles exist? To prove the existence of an ERD, we first analyze its properties.

Remark 1. According to Equation (2) and Equation (3), we can see that the concept of ERD is closely related to the concept of stochastic user equilibrium (SUE). In particular, an ERD satisfies SUE condition [17] in Equation (4).

$$
f^{\nu, i}=p^{\nu, i}\left(f_{h^{i}}\right) \cdot 1, \forall, \nu=1, \ldots, m ; i=1, \ldots, k^{\nu} .
$$

Generally speaking, this observation implies that if an ERD is reached, then an equilibrium expected route flow in the near future is reached. However, the ERD concept and the SUE concept are proposed for completely different purposes. The SUE concept is for traffic planning purpose, and hence focus on macroscopic measures of the traffic network, such as link traffic flow, link travel time, etc, while the ERD concept is for individual vehicle route choice decision, and hence focus on individual vehicles' route choices. 


\subsection{Existence and uniqueness of the ERD}

This section explores existence and uniqueness of ERD. To do this, we rely on a mathematical programming (MP) given below. We will show that (MP) has a nice property that its optimal solution set coincides with the ERD set. This idea can be traced back to [6].

$$
\begin{aligned}
(\mathrm{MP}): \min Z(f) & =\sum_{\ell \in A} \int_{0}^{f_{\ell}} c_{\ell}(\omega) d \omega+\sum_{\nu=1}^{m} \sum_{i=1}^{k^{\nu}} \frac{1}{\beta^{\nu}} f^{\nu, i} \ln f^{\nu, i}+\sum_{\nu=1}^{m} \sum_{i=1}^{k^{\nu}} \frac{\alpha^{\nu}}{\beta^{\nu}} f^{\nu, i} \\
1 & =\sum_{i=1}^{k^{\nu}} f^{\nu, i}, \forall \nu=1, \cdots, m \\
f_{\ell} & =\sum_{\nu=1}^{m} \sum_{i=1}^{k^{\nu}} f^{\nu, i} \delta_{\nu, i}^{\ell}, \forall \ell=1, \cdots, l \\
f^{\nu, i} & \geq 0
\end{aligned}
$$

where, $f^{\nu, i}, \forall \nu=1, \cdots, m, i \in \kappa^{\nu}$ represents the decision variables; The constraints are standard flow conservation constraints. To make sure the objective function of (MP) is well defined on the whole feasible region, we let $f^{\nu, i} \ln f^{\nu, i}=0$ if $f^{\nu, i}=0$, then (MP) has a continuous objective function since

$$
\lim _{f^{\nu, i} \rightarrow 0} f^{\nu, i} \ln f^{\nu, i}=0 .
$$

We can show that the optimal solution of (MP) has the properties stated in Lemma 2 and Lemma 4.

Lemma 2. Let $f=\left(f_{\ell}, f^{\nu, i}\right)_{\ell=1, \cdots, l, \nu=1, \cdots, m, i=1, \cdots, k^{\nu}}$ be a local minimizer of (MP). It holds that $f^{\nu, i}>0$ for all $\nu=1, \cdots, m, i=1, \cdots, k^{\nu}$.

Proof. For the sake of contradiction, suppose there is a local minimizer $f$ such that $f^{\nu^{*}, i^{*}}=0$ for some vehicle $\nu^{*} \in\{1, \cdots, m\}$ and its path $i^{*} \in\left\{1, \cdots, k^{\nu^{*}}\right\}$. Since $\sum_{i=1}^{k^{\nu}} f^{\nu, i}=1$, there must exist a $j^{*} \neq i^{*}$ such that $f^{\nu^{*}, j^{*}}=b>0$. Now for any small positive $\varepsilon>0$, we construct another solution $\bar{f}$ as follows

$$
\begin{aligned}
\bar{f}^{\nu^{*}, i^{*}} & =f^{\nu^{*}, i^{*}}+\varepsilon, \\
\bar{f}^{\nu^{*}, j^{*}} & =f^{\nu^{*}, j^{*}}-\varepsilon, \\
\bar{f}^{\nu^{*}, i} & =f^{\nu^{*}, i}, \forall i=1, \cdots, k^{\nu^{*}}, i \neq i^{*}, i \neq j^{*}, \\
\bar{f}^{\nu, i} & =f^{\nu, i}, \forall \nu \neq \nu^{*}, i=1, \cdots, k^{\nu}, \\
\bar{f}_{\ell} & =\sum_{\nu=1}^{m} \sum_{i=1}^{k^{\nu}} \bar{f}^{\nu, i} \delta_{\ell}^{\nu, i} .
\end{aligned}
$$

We compare $Z(f)$ and $Z(\bar{f})$ below.

$$
\begin{aligned}
& Z(\bar{f})-Z(f)=\sum_{\ell \in A} \int_{0}^{\bar{f}_{\ell}} c_{\ell}(\omega) d \omega+\sum_{\nu=1}^{m} \sum_{i=1}^{k^{\nu}} \frac{1}{\beta^{\nu}} \bar{f}^{\nu, i} \ln \bar{f}^{\nu, i}-\sum_{\ell \in A} \int_{0}^{f_{\ell}} c_{\ell}(\omega) d \omega-\sum_{\nu=1}^{m} \sum_{i=1}^{k^{\nu}} \frac{1}{\beta^{\nu}} f^{\nu, i} \ln f^{\nu, i}
\end{aligned}
$$

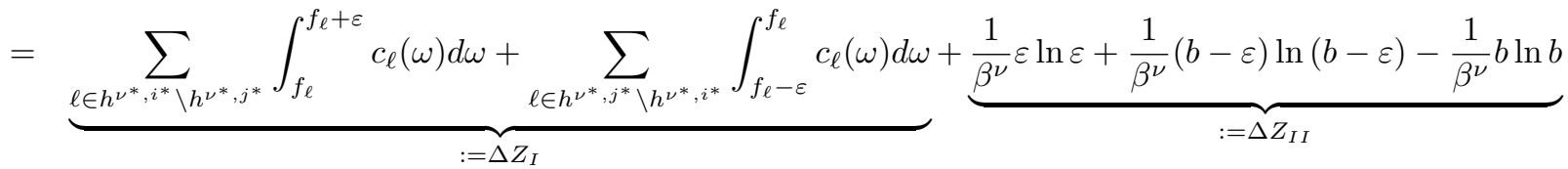

By Assumption (A), we must have $c_{\ell}\left(f_{\ell}\right)$ is bounded on $\left[f_{\ell}, f_{\ell}+\varepsilon\right]$ for all $\ell \in A$, therefore we have there exists an $M_{1}$ such that $c_{\ell}\left(f_{\ell}\right)<M_{1}$ on $\left[f_{\ell}, f_{\ell}+\varepsilon\right]$ for all $\ell \in A$. Hence, we obtain an upper bound for $\Delta Z_{I}$ 
below.

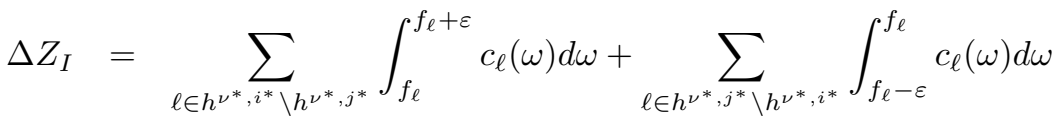

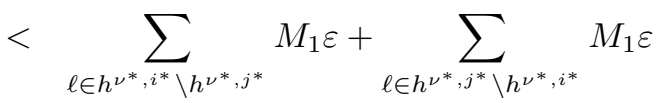

$$
\begin{aligned}
& <2 M_{1} l \varepsilon \text {, }
\end{aligned}
$$

where $l$ is the number of links in $G$. We next consider $\Delta Z_{I I}$. Without loss of generality, we consider each $\varepsilon<\frac{1}{2} b$. The function $x \ln x$ is differentiable on $[b-\epsilon, b]$, and hence by mean value theorem, we know that

$$
\frac{1}{\beta^{\nu}}(b-\varepsilon) \ln (b-\varepsilon)-\frac{1}{\beta^{\nu}} b \ln b=\frac{1}{\beta^{\nu}}\left(1+\ln y_{\varepsilon}\right) \varepsilon,
$$

for some $y_{\varepsilon} \in[b-\epsilon, b]$. Since $\varepsilon<\frac{1}{2} b$, we must also have $y_{\varepsilon} \in\left[\frac{1}{2} b, b\right]$, and then $\left|\ln y_{\epsilon}\right|$ must be bounded by a large enough number $M_{2}$, i.e., $\left|\ln y_{\varepsilon}\right|<M_{2}$, for each $\varepsilon<\frac{1}{2} b$. This implies that

$$
\begin{aligned}
\Delta Z_{I I} & =\frac{1}{\beta_{\nu}} \varepsilon \ln \varepsilon+\frac{1}{\beta^{\nu}}(b-\varepsilon) \ln (b-\varepsilon)-\frac{1}{\beta^{\nu}} b \ln b \\
& <\frac{1}{\beta^{\nu}} \varepsilon \ln \varepsilon+\frac{1}{\beta^{\nu}}\left(1+M_{2}\right) \varepsilon
\end{aligned}
$$

Combining (9) and (10) we obtain:

$$
\begin{aligned}
Z(\bar{f})-Z(f) & =\Delta Z_{I}+\Delta Z_{I I} \\
& <2 M_{1} l \varepsilon+\frac{1}{\beta^{\nu}} \varepsilon \ln \varepsilon+\frac{1}{\beta^{\nu}}\left(1+M_{2}\right) \varepsilon \\
& <\left(2 M_{1} l+\frac{1}{\beta^{\nu}}\left(1+M_{2}\right)+\frac{1}{\beta^{\nu}} \ln \varepsilon\right) \varepsilon
\end{aligned}
$$

for all $\varepsilon<\frac{1}{2} b$. Now for all

$$
\varepsilon<\min \left(\frac{1}{2} b, e^{-2 \beta^{\nu} M_{1} l-1-M_{2}}\right)
$$

we must have

$$
2 M_{1} l+\frac{1}{\beta^{\nu}}\left(1+M_{2}\right)+\frac{1}{\beta^{\nu}} \ln \varepsilon<0,
$$

or equivalently

$$
Z(\bar{f})-Z(f)<0 .
$$

This contradicts the assumption that $\left(f_{\ell}, f^{\nu, i}\right)_{\ell=1, \cdots, l, \nu=1, \cdots, m, i=1, \cdots, k^{\nu}}$ is a local minimizer of (MP). This concludes the proof.

Remark 3. Lemma 2 tells us that if (MP) has a local optimal solution, then this solution can not locate at the relative boundary of the feasible region. As we will see, this is an important technical lemma, especially since the objective function is not differentiable on the relative boundary. This result needs to be established first before the KKT conditions can be applied to a local minimizer of (MP). In the transportation research literature, even though optimization problems similar to (MP) have been widely used for studying SUE problems, a step to establish results similar to Lemma 2 is often missing. We included this proof here for completeness.

We then apply Lemma 2 to show the uniqueness of a global optimal solution to (MP).

Lemma 4. Optimization problem (MP) possesses a unique global optimal solution. 
Proof To proof this Lemma, we eliminate variables $f_{\ell}$ by substituting (8) into the objective function. By doing so, (MP) becomes an optimization problem with constraints (7) and (8), and objective function as follows:

$$
\sum_{\ell=1}^{l} \int_{0}^{\sum_{\nu=1}^{m} \sum_{i=1}^{k^{\nu}} f^{\nu, i} \delta_{\nu, i}^{\ell}} c_{\ell}(\omega) d \omega+\sum_{\nu=1}^{m} \sum_{i=1}^{k^{\nu}} \frac{1}{\beta^{\nu}} f^{\nu, i} \ln f^{\nu, i}+\sum_{\nu=1}^{m} \sum_{i=1}^{k^{\nu}} \frac{\alpha^{\nu}}{\beta^{\nu}} f^{\nu, i} .
$$

Note that we have extended the definition of $f^{\nu, i} \ln f^{\nu, i}$ in a continuous fashion onto the boundaries of the feasible region defined by (7) and (8). Therefore the objective function represented by (11) is continuous. And the feasible region scoped by linear constratins is compact, hence there must exist a global optimal solution. To show the uniqueness, we first show that (11) is strictly convex in the interior of the feasible region defined by (7) and (8). Notice that (11) is in fact twice continuously differentiable in the interior of the feasible region, and the Hessian matrix, denoted by $H(Z)$ is given by

$$
\begin{aligned}
& H(Z)=\Omega\left[\begin{array}{ccccc}
c_{1}^{\prime}\left(\sum_{i=1}^{k^{\nu}} f^{\nu, i} \delta_{\nu, i}^{1}\right) & & & & \\
& \ddots & & & \\
& & c_{\ell}^{\prime}\left(\sum_{i=1}^{k^{\nu}} f^{\nu, i} \delta_{\nu, i}^{\ell}\right) & & \\
& & \ddots & \\
& & & c_{l}^{\prime}\left(\sum_{i=1}^{k^{\nu}} f^{\nu, i} \delta_{\nu, i}^{l}\right)
\end{array}\right] \Omega^{T}
\end{aligned}
$$

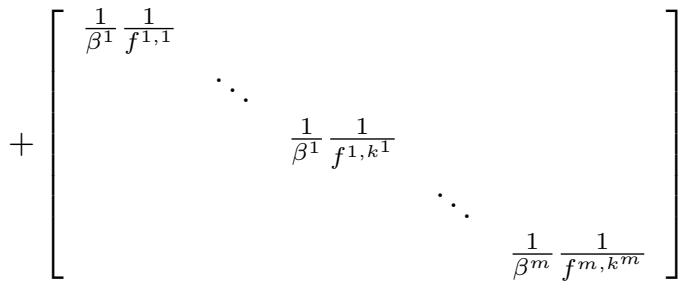

Under Assumption (A), it is easy to prove the first part of $H(Z)$ is positive semi-definite ${ }^{4}$. The second part of $H(z)$ is a diagonal matrix with positive elements so it is a positive definite matrix. Therefore, $H(Z)$ is positive definite. This, together with Lemma 2, implies that that (MP) has unique global optimal solution.

Applying Lemma 2, we further demonstrate the equivalence of ERD and the optimal solution of (MP) in Theorem 5 below.

Theorem 5. The optimal solution of the mathematical programming (MP) is equivalent to the traffic flow satisfying ERD condition in Equation (4).

Proof: $(\Rightarrow)$ We first prove that the optimal solution of (MP) satisfies ERD condition in Equation (4). Since (MP) has only linear constraints, therefore constraint qualification holds. Hence, given an optimal solution of (MP), $\left(f_{\ell}, f^{\nu, i}\right)_{\ell=1, \cdots, l ; \nu=1, \cdots, m ; i=1, \cdots, k^{\nu}}$, we must have the following Karush-Kuhn-Tucker (KKT) conditions hold:

$$
\begin{aligned}
f^{\nu, i}\left(C^{\nu, i}+\frac{1}{\beta^{\nu}}\left(1+\ln f^{\nu, i}\right)+\frac{\alpha^{\nu}}{\beta^{\nu}}-\pi^{\nu}\right) & =0, \forall \nu=1, \cdots, m ; i=1, \cdots, k^{\nu} \\
\sum_{i=1}^{k^{\nu}} f^{\nu, i}-1 & =0, \forall \nu=1, \cdots, m, \\
C^{\nu, i}+\frac{1}{\beta^{\nu}}\left(1+\ln f^{\nu, i}\right)+\frac{\alpha^{\nu}}{\beta^{\nu}}-\pi^{\nu} & \geq 0, \forall \nu=1, \cdots, m ; i=1, \cdots, k^{\nu} \\
f^{\nu, i} & \geq 0, \forall \nu=1, \cdots, m, i=1, \cdots, k^{\nu}
\end{aligned}
$$

\footnotetext{
${ }^{4}$ Given any diagonal matrix $\Lambda=\left[c_{i i}\right], c_{i i} \geq 0$, for any vector $x$ and matrix $M$ of compatible sizes, we must have $x^{T} M \Lambda M^{T} x=$ $\left(M^{T} x\right)^{T} \Lambda\left(M^{T} x\right) \geq 0$. Hence, $M \Lambda M^{T}$ is semi-positive definite.
} 
where $\pi^{\nu}$ is the multiplier of the first constraint; and $C^{\nu, i}$ represent the travel time of path $h^{\nu, i}$. It can be obtained by doing the first-order derivative for the first item in the objective function of (MP). The mathematical process is given below. Note that $f_{\ell}$ is substituted by $f^{\nu, i}$ according to Equation (8).

$$
C^{\nu, i}=\frac{d\left(\sum_{\ell \in A} \int_{0}^{f_{\ell}} c_{\ell}(\omega) d \omega\right)}{d\left(f^{\nu, i}\right)}=\sum_{\ell \in A} c_{\ell}\left(f_{\ell}\right) \frac{d\left(f_{\ell}\right)}{d\left(f^{\nu, i}\right)}=\sum_{\ell \in A} c_{\ell}\left(f_{\ell}\right) \delta_{\nu, i}^{\ell} .
$$

For the complementary slackness in Equation (13), we have $f^{\nu, i}>0$, according to Lemma 2. Furthermore, we have

$$
C^{\nu i}+\frac{1}{\beta^{\nu}}\left(1+\ln f^{\nu, i}\right)+\frac{\alpha^{\nu}}{\beta^{\nu}}=\pi^{\nu} .
$$

Rearrange Equation (18), we have

$$
f^{\nu, i}=e^{\beta^{\nu} \pi^{\nu}-1} \cdot e^{-\left(\alpha^{\nu}+\beta^{\nu} C^{\nu, i}\right)} .
$$

Combining Equation (19) and Equation (14), we obtain

$$
f^{\nu, i}=\frac{e^{-\left(\alpha^{\nu}+\beta^{\nu} C^{\nu, i}\right)}}{\sum_{i=1}^{k^{\nu}} e^{-\left(\alpha^{\nu}+\beta^{\nu} C^{\nu, i}\right)}}=p^{\nu, i}, \forall \nu=1, \cdots, m ; i=1, \cdots, k^{\nu} .
$$

Thus, it proves that the optimal solution of (MP) satisfies ERD condition in Equation (4).

$(\Leftarrow)$ The above proof also indicates that the traffic flow satisfying ERD condition in Equation (4) will satisfy KKT conditions from Equations (13) to (15). This completes the proof of Theorem 5.

The above theorem demonstrates the existence of an ERD for the proposed coordinated mixed strategy routing game. This study next prove uniqueness of the ERD in theorem 7 .

Remark 6. Theorem 5 also shows that the objective function of (MP) (Equation 6) is a potential function of the mixed strategy routing game. We will utilize this potential function to analyze the convergence of the distributed algorithm proposed later.

Theorem 7. The proposed mathematical programming (MP) has a unique optimal solution, hence, the proposed coordinated routing mechanism has a unique ERD traffic flow.

Proof Theorem 5 and Equation (4) concludes this theorem without further proof.

\section{Distributed Algorithm}

The study proposes a simultaneously updating distributed algorithm (SDA) to implement the proposed CRM. Figure 1 illustrates the concept of the distributed algorithm in a coordination routing group. Namely, the routing decision negotiation process is performed iteratively with each iteration including three key steps. First, individual smart vehicles locally evaluate traffic condition in the near future according to the latest traffic information including the route choice priorities of other vehicles. Next, smart vehicles update their route choice priorities and propose to the online coordination routing environment for next round of negotiation. Third, the online communication platform aggregates real-time traffic condition and disseminates to all smart vehicles in the coordination routing group with privacy protection. The negotiation process, composed of iterative traffic condition evaluating, route choice priorities updating/proposing, and traffic condition aggregating process, goes on until an ERD is reached among all smart vehicles in the group. Table 1 demonstrates the steps that individual vehicles perform in the SDA. Note that route choice priority of individual smart vehicle $\nu$ is updated following the equations below:

$$
p_{t+1}^{\nu}=p_{t}^{\nu}+\lambda_{t} d^{\nu}
$$

where the moving direction is decided by

$$
d^{\nu}=\left(p_{t+1}^{\nu, \rightarrow}-p_{t}^{\nu}\right)
$$


and

$$
p_{t+1}^{\nu, \rightarrow}=\left(p_{t+1}^{\nu, i, \rightarrow}\right)_{i=1, \cdots, k^{\nu}},
$$

whose component are defined by (3). The SDA simultaneously generates real-time routing guidance for all individual vehicles, integrating an underlying route choice decision collaboration among them.

The applicability of the SDA for the coordinated online in-vehicle routing relies on the answers to two questions: (1) will the SDA converge to an ERD; (2) how fast the SDA can converge? This study provides rigorous proof for the convergence of the SDA in the next section and discuss its convergence rate through case study provided in Section 5 .

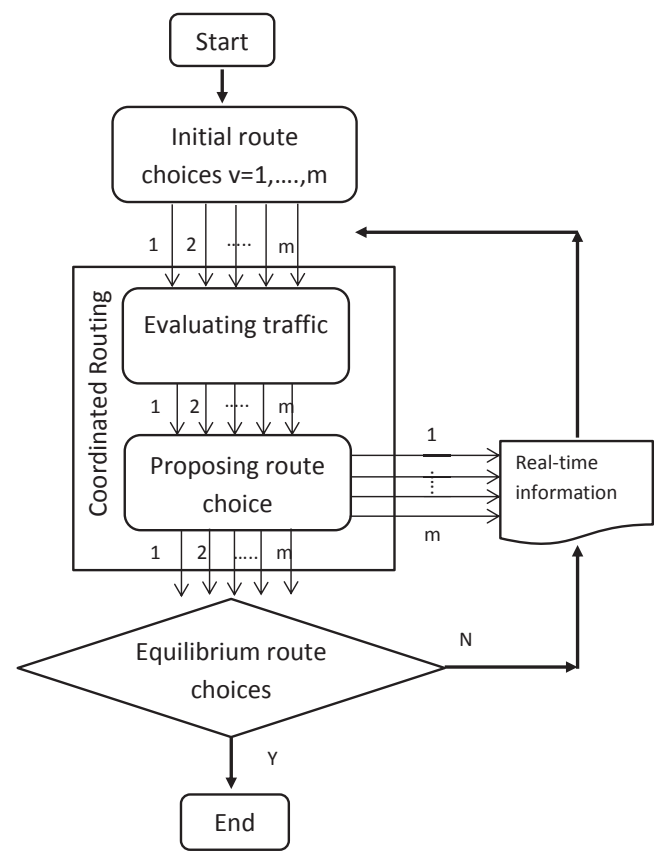

Figure 1: The flow chart of the distributed algorithm for the CRM

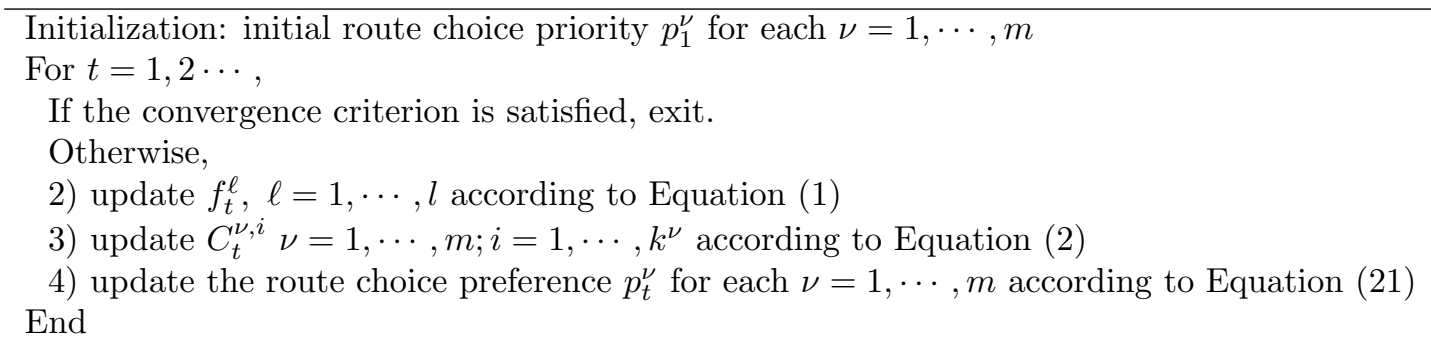

Table 1: Distributed algorithm for multiple vehicle route choice decision

Remark 8. In this algorithm, the initialization step and step 4) are performed simultaneously by the individual vehicles, step 2) and 3) are performed by either a central communication facility or individual vehicles, after the updated route choice preference information is available from the pervious iteration.

\subsection{Existing Theorems}

This study proves the convergence of the SDA by applying a general theorem regarding the convergence of an iterative algorithm appeared in [1]. The proof process also employs Theorem 10 in [1] and the inequality given in Lemma 11. To be self-contained, we provide these existing theorems below. 
Theorem 9. [1] Let $X$ be a nonempty closed set in $\mathbb{R}_{n}$, and let the nonempty set $S \subseteq X$ be the solution set. Let $A: X \rightarrow X$ be a point-to-set map. Given $x_{1} \in X$, the sequence $\left\{x_{k}\right\}$ is generated iteratively as follows: if $x_{k} \in S$ then stop; otherwise, let $x_{k+1} \in A\left(x_{k}\right)$, replace $k$ by $k+1$, and repeat. Suppose the sequence $x_{1}, x_{2}, \ldots$ produced by the algorithm is in a compact set $X$, and suppose that there exists a continuous function $\varphi$, called the decent function, such that $\varphi(y)<\varphi(x)$ if $x \nsubseteq S$ and $y \in A(x)$. If the map $A$ is closed over the complement of $S$, then either the algorithm stops in a finite number of steps with a point in $S$ or it generates the infinite sequence $\left\{x_{k}\right\}$ such that

1. Every convergent subsequence of $\left\{x_{k}\right\}$ has a limit in $S$, that is, all accumulation point of $\left\{x_{k}\right\}$ belong to $S$.

2. $\varphi\left(x_{k}\right) \rightarrow \varphi(x)$ for some $x \in S$.

Theorem 10. [1] Let $X, Y$, and $Z$ be nonempty closed sets in $\mathbb{R}_{n}, \mathbb{R}_{p}$, and $\mathbb{R}_{q}$, respectively. Let $\boldsymbol{B}: X \rightarrow Y$ be a function, and let $\boldsymbol{C}: Y \rightarrow Z$ be a point-to-set map, if $\boldsymbol{B}$ is continuous at $x$ and $\boldsymbol{C}$ is closed on $\boldsymbol{B}(x)$, then $\boldsymbol{A}=\boldsymbol{C B}$ is close at $x$.

Lemma 11. [4] If the vector $q, p>0$ satisfies $\sum_{1}^{n} q_{i}=1$ and $\sum_{1}^{n} p_{i}=1$, then it holds that

$$
\sum_{i}^{n} p_{i} \log \frac{p_{i}}{q_{i}} \geq \frac{1}{2}\left(\sum_{1}^{n}\left|p_{i}-q_{i}\right|\right)^{2} .
$$

Remark 12. Lemma 11 is a key technical lemma that allows us to perform convergence analysis for our distributed algorithm. This inequality has been studied in the information theory field. This paper demonstrate that it can also be applied in the study of transportation problems involving discrete choice models.

To demonstrate the application of Theorem 9, we make the following clarifications for our problem. (i) The set composed of all possible joint route choice priorities for all smart vehicles in a coordination routing group corresponds to set $X$ in Theorem 9 . By the definition of probability, we have $\sum_{i} p^{\nu, i}=1$, and $p^{\nu, i} \geq 0$ for each $\nu$, so this set is a close set. (ii) The ERD represents the solution set $S$ in Theorem 9. According to Theorem 7 , there is only one solution in $S$, which is also the optimal solution of (MP). (iii) The objective function of (MP), which is the potential function of the game, is used as the decent function in Theorem 9. The procedure to prove the convergence of the SDA includes three key steps: (1) prove Theorem 13 which indicates that the potential function of the CRM can be a decent function if certain update step size is chosen; (2) relying on Theorem 10, prove Theorem 15 which demonstrates that the routing decision update method proposed in this study is a closed map; (3) conclude the convergence of the SDA by applying Theorem 9. The following subsections provide the technical details for each step.

\subsection{Decent function and step size}

As we have mentioned, this study uses the potential function, or equivalently, the objective function of (MP) as the decent function, with the link flow variables being substituted by the sum of probabilities of the vehicles taking the paths including this link (see the clarification in Section 3.1 for this equivalence). That is, we let

$$
Z(p)=\sum_{\ell=1}^{l} \int_{0}^{\sum_{\nu=1}^{m} \sum_{i=1}^{k^{\nu}} p^{\nu, i} \delta_{\nu, i}^{\ell}} c_{\ell}(\omega) d \omega+\sum_{\nu=1}^{m} \sum_{i=1}^{k^{\nu}} \frac{1}{\beta^{\nu}} p^{\nu, i} \ln p^{\nu, i}+\sum_{\nu=1}^{m} \sum_{i=1}^{k^{\nu}} \frac{\alpha^{\nu}}{\beta^{\nu}} p^{\nu, i} .
$$

We will show that the direction $d=\left(d^{\nu}\right)_{n u=1, \cdots, m}$ is a decent direction of the potential function. Moreover, we provide a formula to compute a step length such that the decreasing of the potential function is guaranteed as the iteration goes on. This formula will be first presented below and then a theorem will be proved to show why this step length formula works. Recall that the Hessian matrix of $(23)$ is denoted by $H(Z(p))$. We let $H\left(Z\left(p_{t}\right)\right)$ and $H\left(Z\left(p_{t=1}\right)\right)$ be the Hessian matrix of (23) at $p_{t}$ and $\overrightarrow{p_{t+1}}$, respectively. Based on assumption (A) and equation (12), we can see that $H\left(Z\left(p_{t}\right)\right)$ has only nonnegative elements for any $p_{t}>0$, where the inequality is considered element wise. Moreover, according to (21) and (22), we can see that if the initial 
probability vector is element-wise positive, i.e., $p_{0}>0$, then the probability vector is always positive in all the iterations, i.e., $p_{t}>0$, for all $t=0,1, \cdots$. We let the step length at iteration $t$ to be

$$
\lambda_{t}=0.99 * \frac{1}{\max _{\nu}\left\{\beta_{\nu}\right\} \sqrt{\tau} \gamma_{t}|\mathcal{P}|},
$$

where $|\mathcal{P}|$ is the number of all paths, $\tau$ is the potion of nonzero elements in matrix $\Omega \Omega^{T}$, and $\gamma_{t}$ is the largest element of the following matrix:

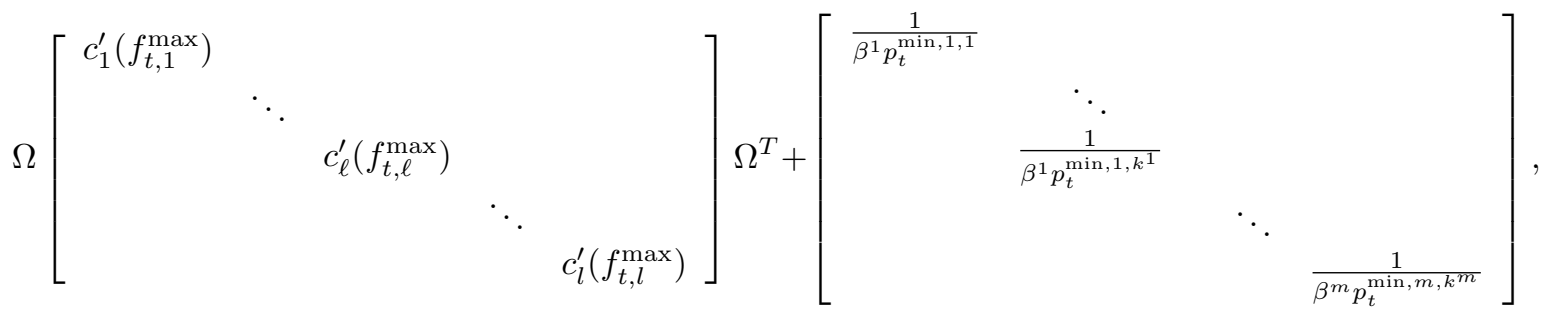

where

$$
\begin{array}{rlrl}
p_{t}^{\min , \nu, i} & =\min \left\{p_{t}^{\nu, i}, p_{t+1}^{\rightarrow, \nu, i}\right\}, & \forall \nu=1, \cdots, m, i=1, \cdots, k^{\nu} \\
p_{t}^{\max , \nu, i}=\max \left\{p_{t}^{\nu, i}, p_{t+1}^{\rightarrow, \nu, i}\right\}, & \forall \nu=1, \cdots, m, i=1, \cdots, k^{\nu} \\
f_{t, \ell}^{\max }=\sum_{\nu=1}^{m} \sum_{i=1}^{k^{\nu}} p_{t}^{\max , \nu, i} \delta_{\nu, i}^{\ell}, & \forall \ell=1, \cdots, l .
\end{array}
$$

Theorem 13. Assume condition (A). Let $\lambda_{t}$ be defined by (24), $d$ be defined by (22), and $p_{t+1}=p_{t}+\lambda_{t} d$. We have if $p_{0}>0$, then $Z\left(p_{t+1}\right)<Z\left(p_{t}\right)$ for all $t=0,1, \cdots$.

Proof. For any iteration $t$, as discussed before, we must have $p_{t}>0$ and $\overrightarrow{t+1}>0$. To prove Theorem 13 we look at Taylor expansion of (23) at $p_{t}$. We have

$$
Z\left(p_{t+1}\right)-Z\left(p_{t}\right)=\lambda_{t} d^{T} \nabla Z\left(p_{t}\right)+\frac{1}{2} \lambda_{t}^{2} d^{T} H\left(Z\left(\bar{p}_{t}\right)\right) d
$$

where $\bar{p}_{t}$ is a vector between $p_{t}$ and $p_{t+1}$, i.e., $\bar{p}_{t}=\mu_{t} p_{t}+\left(1-\mu_{t}\right) p_{t+1}$ for some scalar $\mu_{t}$ satisfying $0 \leq \mu_{t} \leq 1$. Note that $p_{t+1}$ is between $p_{t}$ and $\overrightarrow{p_{t+1}}$, hence $\bar{p}_{t}$ is also between $p_{t}$ and $\overrightarrow{p_{t+1}}$. The gradient of (23) is given by:

$$
\nabla Z(p)=\left(C^{\nu, i}+\beta^{\nu}\left(1+\ln p^{\nu, i}\right)\right)_{\nu=1, \cdots, m ; i=1, \cdots, k^{\nu}},
$$

where

$$
C^{\nu, i}=\sum_{\ell \in h^{\nu, i}} c\left(\sum_{\nu=1}^{m} \sum_{h^{\nu, i} \ni \ell} p^{\nu, i}\right)
$$

which is actually the expected travel time for path $h^{\nu, i}$ of vehicle $\nu$ when the joint probability vector is $p$. Letting $C_{t}^{\nu, i}=\sum_{\ell \in h^{\nu, i}} c\left(\sum_{\nu=1}^{m} \sum_{h^{\nu, i} \ni \ell} p_{t}^{\nu, i}\right)$, we have

$$
\begin{aligned}
& d_{t}^{T} \nabla Z\left(p_{t}\right) \\
& =\sum_{\nu=1}^{m} \sum_{i=1}^{k^{\nu}}\left[\left(p_{t+1}^{\rightarrow, \nu, i}-p_{t}^{\nu, i}\right)\left(C_{t}^{\nu, i}+\frac{1}{\beta^{\nu}}\left(1+\ln p_{t}^{\nu, i}\right)\right)\right] \\
& =\sum_{\nu=1}^{m} \sum_{i=1}^{k^{\nu}}\left[C_{t}^{\nu, i} p_{t+1}^{\rightarrow, \nu, i}-C_{t}^{\nu, i} p_{t}^{\nu, i}+\frac{1}{\beta^{\nu}}\left(1+\ln p_{t}^{\nu, i}\right) p_{t+1}^{\rightarrow, \nu, i}-\frac{1}{\beta^{\nu}} p^{\nu, i}\left(1+\ln p^{\nu, i}\right)\right] \\
& =\sum_{\nu=1}^{m} \sum_{i=1}^{k^{\nu}}\left[C_{t}^{\nu, i} p_{t+1},-C_{t}^{\nu, i} p_{t}^{\nu, i}+\frac{1}{\beta^{\nu}} p_{t+1}^{\rightarrow, \nu, i} \ln p_{t}^{\nu, i}-\frac{1}{\beta^{\nu}} p_{t}^{\nu, i} \ln p_{t}^{\nu, i}\right] \\
& =\sum_{\nu=1}^{m}\left[\sum_{i=1}^{k^{\nu}}\left(C_{t}^{\nu, i} p_{t+1}^{\rightarrow, \nu, i}+\frac{1}{\beta^{\nu}} p_{t+1}^{\rightarrow, \nu, i} \ln p_{t+1}^{\rightarrow, \nu, i}\right)-\sum_{i=1}^{k^{\nu}}\left(C_{t}^{\nu, i} p_{t}^{\nu, i}+\frac{1}{\beta^{\nu}} p_{t}^{\nu, i} \ln p_{t}^{\nu, i}\right)-\frac{1}{\beta^{\nu}} \sum_{i=1}^{k^{\nu}}\left\{p_{t+1}^{\rightarrow, \nu, i} \ln \frac{p_{t+1}^{\rightarrow, \nu, i}}{p_{t}^{\nu, i}}\right\}\right]
\end{aligned}
$$


Note that $\left(p_{t+1}^{\rightarrow, \nu, i}\right)_{i=1, \cdots, k^{\nu}}$ is the solution of the following optimization problem:

$$
\begin{aligned}
\left(p_{t+1}^{\rightarrow, \nu, i}\right)_{i=1, \cdots, k^{\nu}} \in \operatorname{argmin}_{q_{1}, \cdots, q_{k}} & \sum_{i=1}^{k^{\nu}}\left(C_{t}^{\nu, i} q_{t+1}^{i}+\frac{1}{\beta^{\nu}} q_{t+1}^{i} \ln q_{t+1}^{i}\right) \\
\text { s.t. } & \sum_{i=1}^{k^{\nu}} q_{t+1}^{i}=1 \\
& q_{t+1}^{i} \geq 0, \forall i=1, \cdots, k^{\nu}
\end{aligned}
$$

Therefore, we must have

$$
\sum_{i=1}^{k^{\nu}}\left\{C_{t}^{\nu, i} p_{t+1}^{\rightarrow, \nu, i}+\frac{1}{\beta^{\nu}} p_{t+1}^{\rightarrow, \nu, i} \ln p_{t+1}^{\rightarrow, \nu, i}\right\}-\sum_{i=1}^{k^{\nu}}\left\{C_{t}^{\nu, i} p_{t}^{\nu, i}+\frac{1}{\beta^{\nu}} p_{t}^{\nu, i} \ln p_{t}^{\nu, i}\right\} \leq 0
$$

Substituting (29) into (27), we obtain that

$$
d_{t}^{T} \nabla Z\left(p_{t}\right) \leq-\sum_{\nu=1}^{m} \frac{1}{\beta^{\nu}} \sum_{i=1}^{k^{\nu}}\left\{p_{t+1}^{\vec{t}, \nu, i} \ln \frac{\overrightarrow{p_{t+1}, \nu, i}}{p_{t}^{\nu, i}}\right\},
$$

According to the inequality stated in Theorem 11 given by [4], we further observed that

$$
d_{t}^{T} \nabla Z\left(p_{t}\right) \leq-\sum_{\nu=1}^{m} \frac{1}{\beta^{\nu}} \sum_{i=1}^{k^{\nu}}\left\{p_{t+1}^{\rightarrow, \nu, i} \ln \frac{p_{t+1}^{\rightarrow, \nu, i}}{p_{t}^{\nu, i}}\right\} \leq-\sum_{\nu=1}^{m} \frac{1}{2 \beta^{\nu}}\left(\sum_{i=1}^{k^{\nu}}\left|p_{t+1}^{\rightarrow, \nu, i}-p_{t}^{\nu, i}\right|\right)^{2} .
$$

According to Equation (30), $Z\left(p_{t+1}\right)-Z\left(p_{t}\right)$ is further bounded in Equation (31) below

$$
\begin{aligned}
& Z\left(p_{t+1}\right)-Z\left(p_{t}\right) \\
& \leq-\lambda_{t} \sum_{\nu=1}^{m} \frac{1}{2 \beta^{\nu}}\left(\sum_{i=1}^{k^{\nu}}\left|p_{t+1}^{\rightarrow, \nu, i}-p_{t}^{\nu, i}\right|\right)^{2}+\frac{1}{2} \lambda_{t}^{2} d_{t}^{T} H(Z(\bar{p})) d_{t} \\
& \leq-\lambda_{t} \sum_{\nu=1}^{m} \frac{1}{2 \beta^{\nu}} \sum_{i=1}^{k^{\nu}}\left(p_{t+1}^{\rightarrow, \nu, i}-p_{t}^{\nu, i}\right)^{2}+\frac{1}{2} \lambda_{t}^{2} d_{t}^{T} H(Z(\bar{p})) d_{t} .
\end{aligned}
$$

Now we look at the second term of the right-hand side in equation (31). According to the discussion in [3], we know that

$$
\frac{d_{t}^{T} d_{t}}{d_{t}^{T} H\left(Z\left(\bar{p}_{t}\right)\right) d_{t}} \geq \frac{1}{e_{t}}
$$

where $\frac{1}{e_{t}}$ is the reciprocal of the largest eigenvalue of $H\left(Z\left(\bar{p}_{t}\right)\right)$. According to [20], we know that the largest eigenvalue of $H(Z(\bar{p}))$ can be bounded from above, i.e.,

$$
e_{t} \leq \sqrt{\bar{\tau}_{t}} \bar{\gamma}_{t}|\mathcal{P}|,
$$

where $|\mathcal{P}|$ represents the order of $H\left(Z\left(\bar{p}_{t}\right)\right)$, which is the number of all paths here; $\bar{\gamma}_{t}$ is the largest element of $H\left(Z\left(\bar{p}_{t}\right)\right) ; \bar{\tau}_{t}$ represents the proportion of of non-zero elements in $H\left(Z\left(\bar{p}_{t}\right)\right)$. Recall the definition of the $\gamma_{t}$ and $\tau_{t}$, as well as the fact that $\bar{p}_{t}$ is between $p_{t}$ and $\overrightarrow{p_{t+1}}$. We claim that

$$
\bar{\gamma}_{t} \leq \gamma_{t} \text {, and } \bar{\tau}_{t}=\tau \text {. }
$$

In fact, since $\bar{p}_{t}$ is between $p_{t}$ and $\overrightarrow{p_{t+1}}$, by the definitions for $p_{t}^{\max , \nu, i}, f_{t, \ell}^{\max }$, and $p_{t}^{\min , \nu, i}$ in equation (26), we must have

$$
\begin{aligned}
& \bar{p}_{t}^{\nu, i} \leq p_{t}^{\max , \nu, i}, \forall \nu=1, \cdots, m ; i=1, \cdots, k^{\nu} . \\
\Rightarrow & \sum_{\nu=1}^{m} \sum_{i=1}^{k^{\nu}} \bar{p}_{t}^{\nu, i} \delta_{\nu, i}^{\ell} \leq \sum_{\nu=1}^{m} \sum_{i=1}^{k^{\nu}} p_{t}^{\max , \nu, i} \delta_{\nu, i}^{\ell}, \forall \ell=1, \cdots, l . \\
\Rightarrow & \bar{f}_{t, \ell} \leq f_{t, \ell}^{\max }, \forall \ell=1, \cdots, l,
\end{aligned}
$$


where

$$
\bar{f}_{t, \ell}=\sum_{\nu=1}^{m} \sum_{i=1}^{k^{\nu}} \bar{p}_{t}^{\nu, i} \delta_{\nu, i}^{\ell} .
$$

Since $c_{\ell}^{\prime}\left(f_{\ell}\right)$ is increasing for all $\ell=1, \cdots, l$, we have

$$
c_{\ell}^{\prime}\left(\bar{f}_{t, \ell}\right) \leq c_{\ell}^{\prime}\left(f_{t, \ell}^{\max }\right), \forall \ell=1, \cdots, l .
$$

On the other hand, we also have

$$
\frac{1}{\beta^{\nu} \bar{p}_{t}^{\nu, i}} \leq \frac{1}{\beta^{\nu} p_{t}^{\min , \nu, i}}, \forall \nu=1, \cdots, m ; i=1, \cdots, k^{\nu} .
$$

Notice that each row of $\Omega$ corresponds to a path, from (12) we can see that for each element $\bar{z}_{r, s}$ of $H\left(Z\left(\bar{p}_{t}\right)\right)$, there exists $(\nu, i)$ and $\left(\nu^{\prime}, i^{\prime}\right)$ so that

$$
\bar{z}_{r, s}=\left\{\begin{array}{cc}
\sum_{\ell \in h^{\nu, i} \cap h^{\nu^{\prime}, i^{\prime}}} c_{\ell}^{\prime}\left(\bar{f}_{t, \ell}\right) & \text { if } r \neq s \\
\sum_{\ell \in h^{\nu, i}} c_{\ell}^{\prime}\left(\bar{f}_{t, \ell}\right)+\frac{1}{\beta^{\nu} \bar{p}_{t}^{\nu, i}} & \text { if } r=s
\end{array}\right.
$$

Now combine (32), (33), and (34) we obtain that

$$
\bar{z}_{r, s} \leq \gamma_{t},
$$

which in turn implies that $\bar{\gamma}_{t}=\max _{r, s}\left\{\bar{z}_{r, s}\right\} \leq \gamma_{t}$. By (34), we can also derive that an element of $H\left(Z\left(\bar{p}_{t}\right)\right)$ is non-zero if and only if the paths $h^{\nu, i}$ and $h^{\nu^{\prime}, i^{\prime}}$ share some common links. Therefore, the number of nonzero elements in $H\left(Z\left(\bar{p}_{t}\right)\right)$ is the same as the number of nonzero elements in $\Omega \Omega^{T}$, i.e.,

$$
\tau=\bar{\tau}_{t}
$$

Therefore, we obtain that

$$
\frac{d_{t}^{T} d_{t}}{d_{t}^{T} H\left(Z\left(\bar{p}_{t}\right)\right) d_{t}} \geq \frac{1}{e_{t}} \geq \frac{1}{\sqrt{\bar{\tau}_{t}} \bar{\gamma}_{t}|\mathcal{P}|} \geq \frac{1}{\sqrt{\tau} \gamma_{t}|\mathcal{P}|}
$$

which is equivalent to

$$
d_{t}^{T} H\left(Z\left(\bar{p}_{t}\right)\right) d_{t} \leq \sqrt{\tau} \gamma_{t}|\mathcal{P}| d_{t}^{T} d_{t} .
$$

Combining (31) and (35) we derive that

$$
\begin{aligned}
Z\left(p_{t+1}\right)-Z\left(p_{t}\right) & \leq-\lambda_{t} \sum_{\nu=1}^{m} \frac{1}{2 \beta^{\nu}} \sum_{i=1}^{k^{\nu}}\left(p_{t+1}^{\rightarrow, \nu}-p_{t}^{\nu, i}\right)^{2}+\frac{1}{2} \lambda_{t}^{2} d_{t}^{T} H(Z(\bar{p})) d_{t} \\
& \leq-\frac{1}{2} \lambda_{t} \frac{1}{\max _{\nu}\left\{\beta^{\nu}\right\}} d_{t}^{T} d_{t}+\frac{1}{2} \lambda_{t}^{2} \sqrt{\tau} \gamma_{t}|\mathcal{P}| d_{t}^{T} d_{t} \\
& =\frac{1}{2}\left(-\frac{1}{\max _{\nu}\left\{\beta^{\nu}\right\}}+\lambda_{t} \sqrt{\tau} \gamma_{t}|\mathcal{P}|\right) \lambda_{t} d_{t}^{T} d_{t} \\
& =\frac{1}{2}\left(-\frac{1}{\max _{\nu}\left\{\beta^{\nu}\right\}}+0.99 * \frac{1}{\max _{\nu}\left\{\beta_{\nu}\right\} \sqrt{\tau} \gamma_{t}|\mathcal{P}|} \sqrt{\tau_{t}} \gamma_{t}|\mathcal{P}|\right) \lambda_{t} d_{t}^{T} d_{t} \\
& =\frac{1}{2}\left(-0.01 * \frac{1}{\max _{\nu}\left\{\beta_{\nu}\right\}}\right) \lambda_{t} d_{t}^{T} d_{t}<0 .
\end{aligned}
$$

This concludes the proof.

Remark 14. There are a few remarks we want to make here regarding Theorem 13. 
1. It is worthwhile to point out that the step length here is relatively easy to obtain in terms of computation time, compared to line search approach. For distributed algorithms like what we discuss in this paper, line search will require several rounds of synchronization among the vehicles, which is time consuming since they require additional time and resources for communication and aggregation. On the other hand, we could use $1 / t$ as the step length at iteration $t$, but this will only lead to almost sure convergence, while our approach leads to convergence. Moreover, using $1 / t$ as the step length may lead to slow convergence, since the step length is smaller and smaller as the algorithm goes on.

2. It is possible to derive a fixed step length (a step length that are the same for all the iterations), but this kind of step length tends to be too small and the convergence of the algorithm becomes slow.

3. There are rooms for improving this step length. More detailed discussion is expected to be very technical and hence is beyond the scope of this paper. We will discuss this issue in a separate paper.

4. The method used here can also be applied to design parallel algorithms for computing stochastic user equilibria. Lemma 11, and may other inequalities in information theory, can be possibly applied to other SUE related issues.

\subsection{Step 2: the closeness of the map}

Theorem 15. The map from $p_{t}$ to $p_{t+1}$ is a closed map.

Proof: We define the composite map $\mathbf{A}=\mathbf{S B}$, where $\mathbf{B}: p_{t} \rightarrow \overrightarrow{p_{t+1}}$ is defined by equation (3), which is continuous, and $\mathbf{S}: p_{t} \rightarrow p_{t+1}$ represented by equation (21), which is also continuous since $\lambda_{t}$, as defined in (24) is continuous in $p_{t}$. Therefore, according to Theorem 7.3.2 in [1] and its corollary 2, we claim that the map from $\mathcal{P}_{t}$ to $\mathcal{P}_{t+1}$ is a closed map.

\subsection{Step 3: convergence of the $S D A$}

Combining Theorem 9, Theorem 13 and Theorem 15 together indicates that the SDA converges to the unique optimal solution of (MP), which coincides with the ERD in the mixed strategy game. Therefore, we claim that the SDA will converge to the ERD. This closes our analysis of the convergence of the SDA.

\section{Numerical Experiments}

This study conducts numerical experiments to demonstrate the performance of the proposed routing mechanism and the distributed implementation algorithm in the following three aspects. 1) We test the computational load of the SDA for approaching the convergence. 2) We demonstrate the advantages of the coordinated routing mechanism, CRM to pure independent selfish-routing mechanism, IRM for systemoptimality and user-optimality. 3) We investigate the effect of smart vehicle penetration on CRM's vantage and convergence performance.

\subsection{Experiments setup}

The experiments are setup on a test-bed with the topology of Sioux Falls city network including 24 nodes and 76 links. Link travel time is identified by BPR function given in Equation (36).

$$
c_{\ell}\left(f_{\ell}\right)=t_{o}\left(1+\alpha\left(\frac{f_{\ell}}{\mathcal{C}}\right)^{\beta}\right),
$$

where $t_{o}$ represents free flow link travel time; $\mathcal{C}$ represents link capacity; the parameters $\alpha=0.15$ and $\beta=4$. Free flow link travel time $t_{o}$ and link capacity $\mathcal{C}$ are randomly generated in all experiments. Smart vehicles represented by O-D pairs are also randomly generated. Each smart vehicles is assigned $k^{\nu}=2$ or 4 candidate paths found by k-shortest path algorithm developed by [19] based on free flow traffic condition. Smart vehicle' route choice priority represented by a discrete probability is calculated by multinomial logit choice model given in (3), where $\alpha_{\nu}$ and $\beta_{\nu}$ are values in $[0,1]$ and randomly assigned. The SDA is implemented by MATLAB R2013a. The numerical experiments are conducted on the desktop with processor: Intel(R) Xeon(R) CUP E5-2603 0@1.8Ghz 1.8GHz and RAM: 16.0 GB. 


\subsection{Convergence and computational load}

To investigate the convergence and computational load of the SDA, the experiments with ten scenarios are run, in which the volume of smart vehicles in the coordinated routing group increases from 100 to 1000 with the step size 100. Each scenario was run multiple times (over 20 times) to remove the effect of the randomness. The results are presented in Figure 2 and Figure 3. Figure 2 indicates that the SDA converges consistently as different volumes of smart vehicle are involved in the routing coordination group. The experiment results are consistent to our theoretical proof. Figure 3 demonstrates that the necessary iterations for the SDA to converge increase with the size of the routing coordination group. As 1000 smart vehicles are involved, the SDA need about 350 iterations to converge at an ERD. At the mean time Figure 2 indicates that the SDA converges very quickly in the early period (after about 50-100 iterations), then enters a slow-paced district since the convergence condition in the experiments are strictly high (all smart vehicles obtain exactly the same route choice priority in two consecutive iterations). As a relaxed convergence condition is employed, the SDA will converge in less iterations, which also implies less communication load. Therefore, the SDA may converge more quickly and boost its applicability in practice. In addition, this study found that the current step size bounded by (24) is a relative loose bound, which can be further improved so that speeds up the convergence of the SDA. Considering this is not the main focus of the proposed work, we leave the relevant research as a near future work. Overall, we state that the convergence efficiency of the SDA can be further improved to satisfy practical online applications.
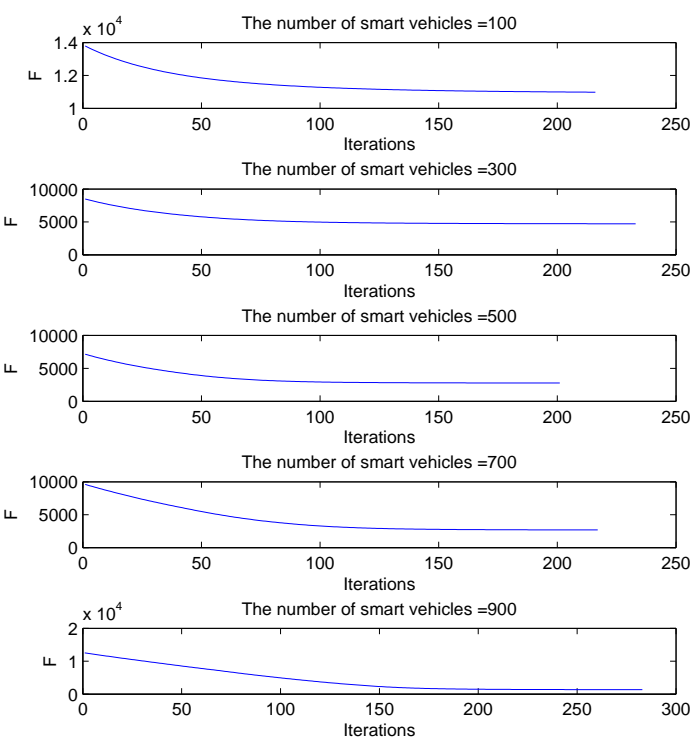
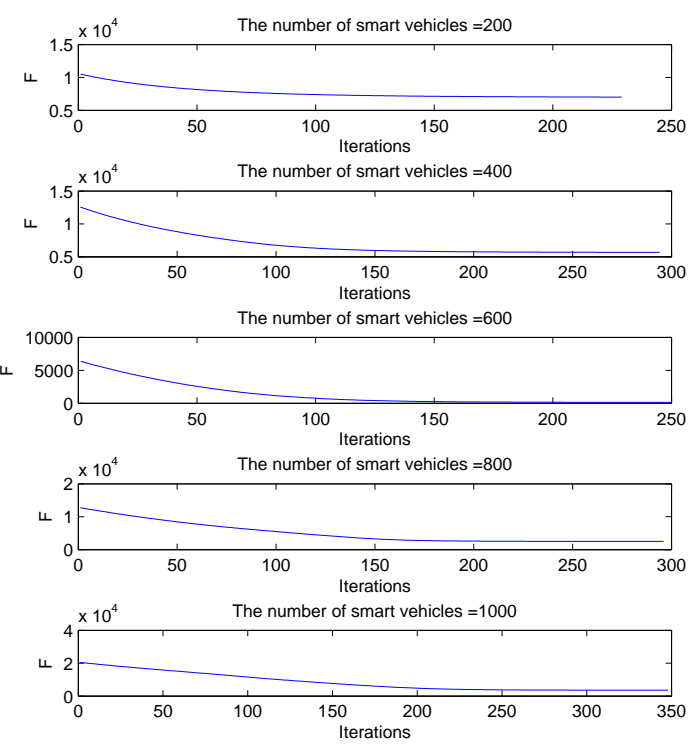

Figure 2: Convergence of CDRM under different smart vehicle fleets.

\subsection{System-optimality and user-optimality}

This study further investigates the advantages of the proposed CRM compared to the traditional IRM (each smart vehicle decides its route choice priority independently without coordination) in both systemoptimality and user-optimality. To do this, we define the system cost and individual vehicle travel cost as $S=\sum_{\ell \in A} c_{\ell} f_{\ell}$ and $T=\sum_{i=1}^{k^{\nu}} C^{\nu, i} p^{\nu, i}$ respectively. Accordingly, the system costs under the CRM and IRM are labeled as $S_{c}$ and $S_{I}$ respectively; average individual vehicle's travel time under the CRM and IRM is labeled as $T_{c}$ and $T_{I}$ respectively. Ten scenarios of experiments are run, in which the volume of smart vehicles in the routing coordination group increases from 100 to 1000 with step size 100, while the total number of vehicles in the system is kept at 1000 (i.e., the smart vehicle penetration increases from 10\% to 100\%). Similarly as before, multiple experiments under each scenario are run to remove the effect of randomness. The performance of the CRM and the IRM are compared in both system-optimality and user-optimality in travel time. 


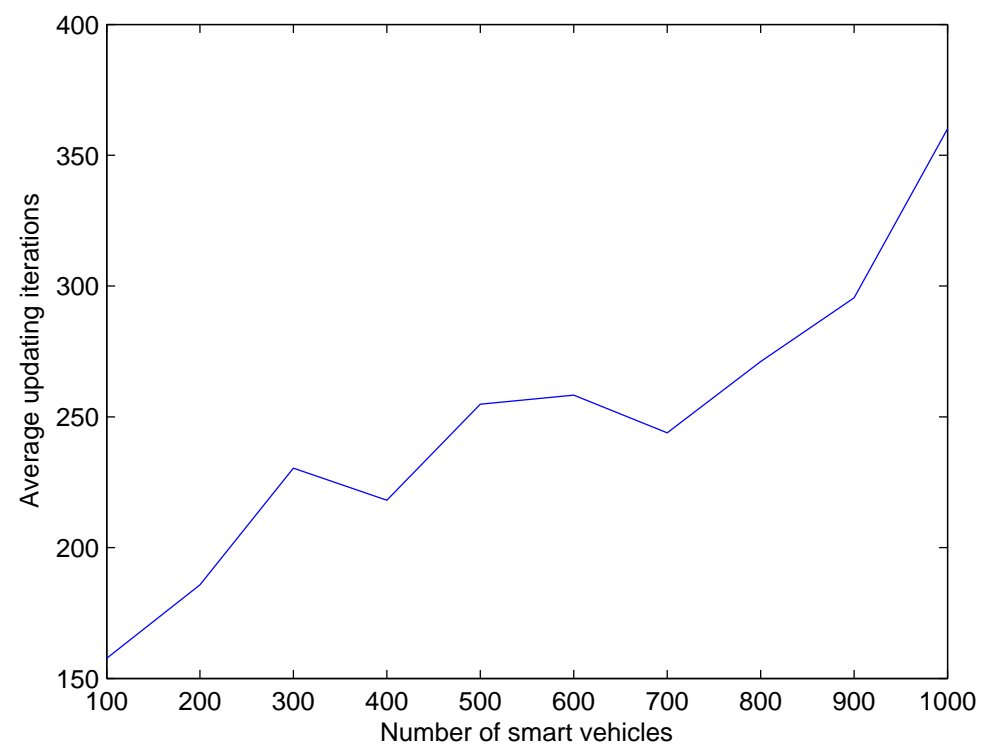

Figure 3: The number of updating iteration for CRM to converge under different smart vehicle fleets.

The experiment results in Figure 4 and Figure 5 indicate that as the penetration of smart vehicles is small (refer to a small size of routing coordination group, such as 100 smart vehicles), the performance of the CRM and the IRM is close to each other (e.g., the ratios, $S_{c} / S_{I}$ to $T_{c} / T_{I}$ in Figure 4 and Figure 5 are almost one); however, as the penetration of smart vehicles increases (e.g., the size of the routing coordination group increases from 100 to 1000 smart vehicles), the advantage of the CRM to the IRM becomes more and more significant (i.e., the ratios of $S_{c} / S_{I}$ to $T_{c} / T_{I}$ in Figure 4 and Figure 5 become smaller and smaller as more smart vehicles are involved. It implies that both system cost and average individual vehicle travel time under the CRM is smaller than under the IRM). Therefore, the proposed CRM outperforms the traditional IRM in both system-optimality and user-optimality.

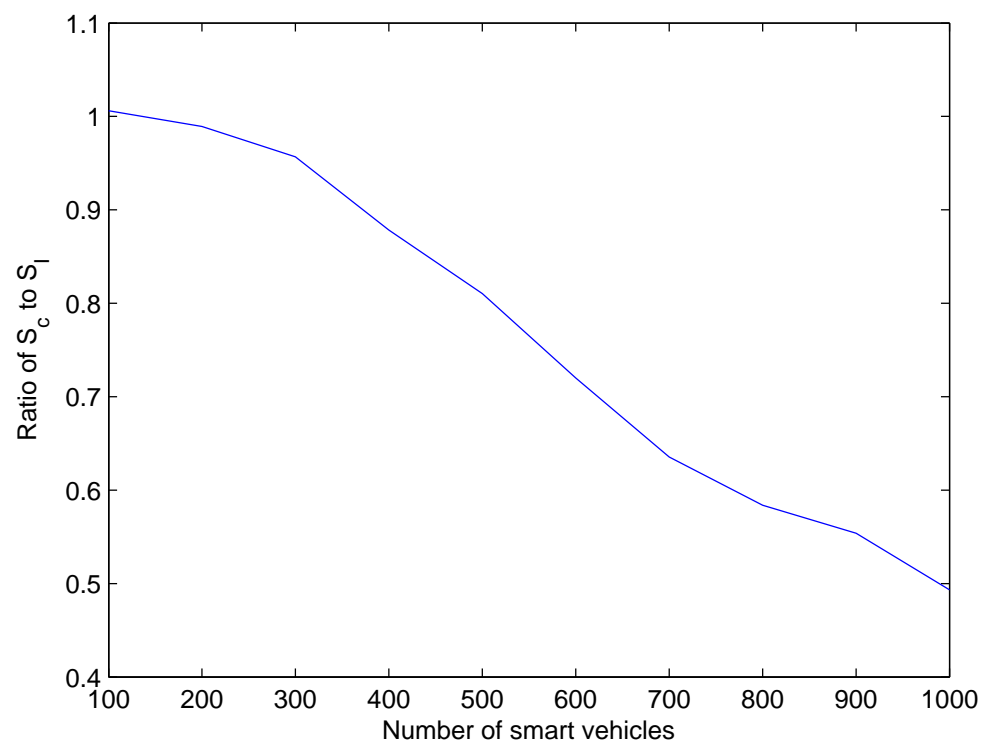

Figure 4: Comparing system cost under the CDRM to that of the IRM. 


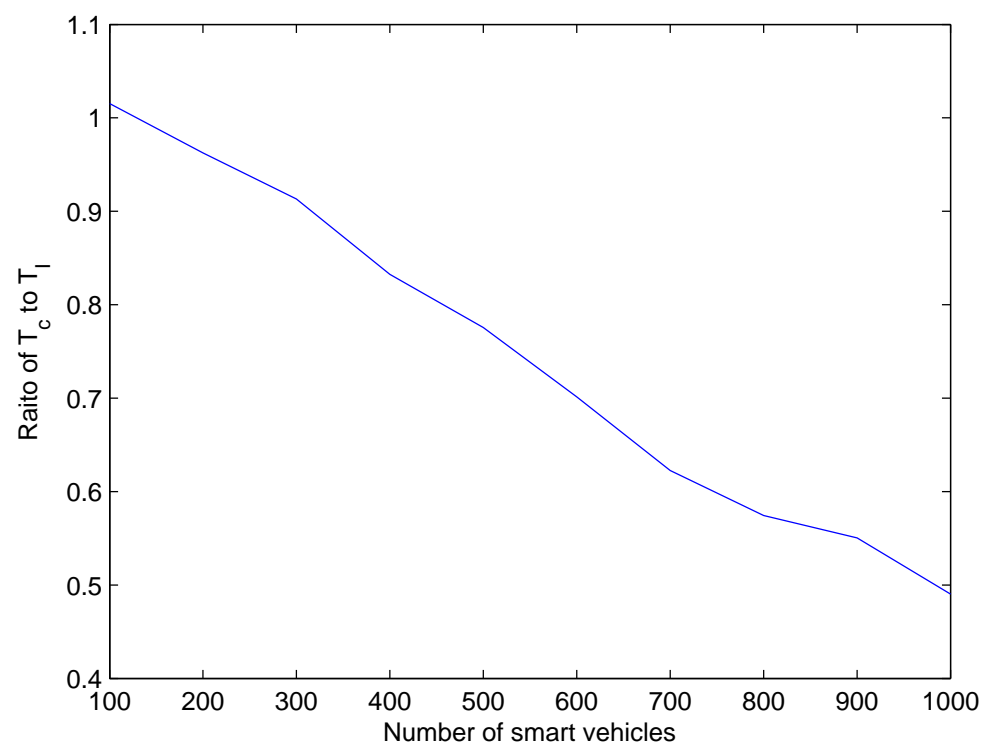

Figure 5: Comparing average individual vehicle travel time under the CDRM to that of the IRM

\section{Conclusion and Future Work}

This research studies the in-vehicle real-time routing guidance for smart vehicle equipped with wireless communication and portable computational facilities. A coordinated online in-vehicle routing mechanism based on a mixed strategy routing game is proposed. It treats online in-vehicle routing as a negotiation and coordination process among smart vehicles, which spontaneously form a routing coordination group according to recent routing decision requests. By following the proposed routing mechanism, smart vehicles decide their online route choice priorities through iteratively proposing and updating their routing choice decisions, responding to real-time traffic information until an equilibrium route choice decision is reached among all smart vehicles. The existence and uniqueness of the equilibrium route choice under the mixed strategy routing game is proved. Furthermore, this study develops a simultaneously updating distributed algorithm to implement the proposed routing mechanism. Its convergence is also proved. The conducted numerical experiments indicate that the proposed simultaneously algorithm converges reasonably quickly under varies smart vehicles penetration. In addition, the proposed coordinated online in-vehicle routing outperforms traditional independent selfish routing in both system cost and individual vehicle cost. It is also noticed that the convergence rate of the proposed distributed algorithms can be further improved as the step size is properly chosen. We propose it as a possible future work.

As we can see, one important technical assumption is Assumption (A). A possible extension of this work is relaxing assumption (A) by allowing the link travel time to be dependent on not only the link flow on itself but also the link flow on other links. This will make the proposed model more realistic but will also introduce significant challenges in designing a good algorithm, especially when monotonicity does not hold. Another possible extension of the work is to introduce traffic dynamics into consideration. This will certainly complicate the problem a lot. One has to carefully balance model realisticity and tractability.

Another assumption in this paper is that traffic information is aggregated and processed perfectly without accuracy and time delay issues. This assumption allows us to perform rigorous mathematical analysis. However, in practice, we need to take into account inaccurate information as well as information time delay. We are conducting research on how imperfect and delayed information can impact the performance of the proposed method in a separate paper.

Acknowledgement: The authors are very grateful to the reviewers for their very constructive comments which have helped us to improve the presentation of this paper. 


\section{References}

[1] Bazaraa, M. S., Sherali, H. D., Shetty, C. M., 2006. Nonlinear Programming: Theory and Algorithms. John Wiley \& Son, Inc.

[2] Ben-Akiva, M., de Palma, A., Kaysi, I., 1996. The impact of predictive information on guidance efficiency: An analytical approach. In: Advanced methods in transportation analysis. Springer, pp. 413432.

[3] Bénasséni, J., 2011. Lower bounds for the largest eigenvalue of a symmetric matrix under perturbations of rank one. Linear and Multilinear Algebra 59 (5), 565-569.

[4] Borwein, J. M., Lewis, A. S., 2006. Convex Analysis and Nonlinear Optimization: Theory and Examples. Vol. 3. Springer.

[5] Du, L., Peeta, S., Kim, Y. H., 2013. Online stochastic routing incorporating real-time traffic information. In: Transportation Research Board 92nd Annual Meeting. No. 13-1477.

[6] Fisk, C., 1980. Some developments in equilibrium traffic assignment. Transportation Research Part B: Methodological 14 (3), 243-255.

[7] Fu, L., 2001. An adaptive routing algorithm for in-vehicle route guidance systems with real-time information. Transportation Research Part B: Methodological 35 (8), 749-765.

[8] Jahn, O., Möhring, R. H., Schulz, A. S., Stier-Moses, N. E., 2005. System-optimal routing of traffic flows with user constraints in networks with congestion. Operations Research 53 (4), 600-616.

[9] Jung, T., Mao, X., Li, X.-Y., Tang, S., Gong, W., Zhang, L., 2013. Privacy-preserving data aggregation without secure channel: Multivariate polynomial evaluation. In: INFOCOM. IEEE, pp. 2634-2642.

[10] Kaufman, D. E., Smith, R. L., Wunderlich, K. E., 1991. An iterative routing/assignment method for anticipatory real-time route guidance. In: Vehicle Navigation and Information Systems Conference, 1991. Vol. 2. IEEE, pp. 693-700.

[11] Kaysi, I., Ben-Akiva, M., Koutsopoulos, H., 1993. Integrated approach to vehicle routing and congestion prediction for real-time driver guidance. Transportation Research Record (1408).

[12] Kim, S., Lewis, M. E., White III, C. C., 2005. Optimal vehicle routing with real-time traffic information. Intelligent Transportation Systems, IEEE Transactions on 6 (2), 178-188.

[13] Miller-Hooks, E., 2001. Adaptive least-expected time paths in stochastic, time-varying transportation and data networks. Networks 37 (1), 35-52.

[14] Miller-Hooks, E. D., Mahmassani, H. S., 2000. Least expected time paths in stochastic, time-varying transportation networks. Transportation Science 34 (2), 198-215.

[15] Papageorgiou, M., 1990. Dynamic modeling, assignment, and route guidance in traffic networks. Transportation Research Part B: Methodological 24 (6), 471-495.

[16] Pretolani, D., 2000. A directed hypergraph model for random time dependent shortest paths. European Journal of Operational Research 123 (2), 315-324.

[17] Sheffi, Y., 1985. Urban transportation networks: Equilibrium analysis with mathematical programming methods.

[18] Stier-Moses, N. E., 2004. Selfish versus coordinated routing in network games. Ph.D. thesis, Massachusetts Institute of Technology.

[19] Yen, J. Y., 1971. Finding the k shortest loopless paths in a network. Management Science 17 (11), $712-716$.

[20] Zhan, X., 2005. Extremal eigenvalues of real symmetric matrices with entries in an interval. SIAM journal on matrix analysis and applications 27 (3), 851-860. 